\title{
Pro und Kontra: Separierte Versorgung gerontopsychiatrischer Patienten
}

\author{
For and Against: Separation of Care for Elderly Patients in Psychiatry
}

Pro

Gabriela Stoppe

In Anbetracht des Alterns der Bevölkerung wird die Krankenversorgung letztendlich insgesamt geriatrisiert. Jedes Fach - wie hier die Psychiatrie - muss Antworten auf diese Entwicklung finden. In dieser Diskussion sind sowohl wissenschaftliche und empirische als auch politische Argumente zu berücksichtigen.

Dabei soll gleich am Anfang gesagt werden, dass die Sicht der Patienten bis heute nicht untersucht wurde. Diese Versorgungsforschungslücke gilt es zu schließen. Wollen zum Beispiel Bipolar-, Schizophrenie- oder auch Suchtkranke mit Krankheitsbeginn im jüngeren und mittleren Lebensalter nach dem Erreichen des 65. Lebensjahres separiert gerontopsychiatrisch behandelt werden? Wollen Ältere vielleicht lieber mit Jüngeren zusammen behandelt werden und wollen diese das auch? Können diagnose- bzw. behandlungsspezifische Abteilungen über alle Altersgruppen hinweg behandeln und sind die Ergebnisse vielleicht sogar besser? Sollen die gesellschaftlich definierten Altersgrenzen (Volljährigkeit versus Ruhestand) auch Grenzen zwischen medizinischen Versorgungsbereichen definieren? Diese Fragen sind bis heute ebenso unbeantwortet, wie hirnorganisch veränderte jüngere Patienten nicht gefragt wurden, ob sie zur Pflege in die Gerontopsychiatrie verlegt werden wollen.

\section{Spezialisiertes Fachwissen}

Wie Untersuchungen zeigen, handelt es sich bei der Mehrheit gerontopsychiatrischer stationärer Patienten um erstmals im Senium erkrankte Menschen [1]. Typisch für psychisch kranke alte Menschen ist eine hohe Komorbidität mit somatischen Erkrankungen. Dies betrifft einerseits hirnorganische Erkrankungen, zum anderen kardiovaskuläre, gastrointestinale und Schmerzerkrankungen. Dies bedeutet einen engeren Austausch mit den somatischen Fächern. Dem trägt z.B. die Schweizer Weiterbildungsordnung Rechnung, die angehenden Geriatern (Schwerpunkt in der Inneren Medizin) eine Pflichtweiterbildungszeit in der Gerontopsychiatrie abverlangt und vice versa den zukünfti- gen Gerontopsychiatern (ab 1.7.06 Schwerpunkt in der Psychiatrie und Psychotherapie) eine Weiterbildungszeit in der somatischen Medizin. Der hier geforderten besonderen Interdisziplinarität und Kompetenz tragen aber auch die an verschiedenen Orten (z. B. Hamburg, Nürnberg) aufgebauten Zentren für Altersmedizin Rechnung. Die enge interdisziplinäre Zusammenarbeit bewährt sich und - soweit schon Ergebnisse vorliegen - zeigt bessere Ergebnisse als monodisziplinäre Ansätze. Dies wird z.B. im Bereich der ambulanten und stationären Demenzversorgung beschrieben $[2,3]$.

Eine spezielle Kompetenz braucht es andererseits im Bereich der Entwicklungspsychologie: das Wissen über die psychische Entwicklung im höheren Lebensalter, Kenntnisse von Kompetenzmodellen oder Copingstrategien. Hier gilt es das in den letzten Jahren erfreulich angewachsene Wissen zu integrieren. Dies hat Einflüsse für die Psychotherapie im höheren Lebensalter aber auch für Sozial- und Milieutherapien [4]. Weitere Spezialkompetenzen können hier nicht ausführlich diskutiert werden. Beispielhaft seien noch Kenntnisse aus der Chronobiologie zu nennen. Der therapeutische Umgang mit (fehlenden) psychosozialen Zeitgebern im Alter ist nicht nur für die Behandlung von Schlafstörungen von Relevanz.

\section{Spezielle Versorgungsstrukturen}

Die Gerontopsychiatrie beinhaltet spezialisierte Versorgungsstrukturen. Die Angebote der Altenhilfe müssen mit denen des medizinischen Sektors in einem Case- bzw. Care-Management verknüpft werden. Schon lange werden hierzu mehr gerontopsychiatrische Zentren gefordert, die stationäre Einrichtungen mit Tageskliniken und Altenberatungsstellen verbinden. Zudem werden alte psychisch kranke Patienten eben oft nicht von Fachärzten versorgt, sondern von ihren Hausärzten. Das sozialpsychiatrische Versorgungssystem ist somit in der Gerontopsychiatrie ein anderes als für andere Altersgruppen.

Zusammengefasst hat die Gerontopsychiatrie heute nicht nur eigene spezialisierte wissenschaftliche Erkenntnisse, sondern auch andere Partner im Gesundheitswesen. Diesem Spezialwis- 
sen kann und sollte eine gerontopsychiatrische Spezialisierung Rechnung tragen.

\section{Ergebnisse und Defizite}

Ob nun eine Separierung bessere Ergebnisse bringt, haben wir selbst untersucht. So werteten wir retrospektiv Krankengeschichten der Psychiatrischen Universitätsklinik Göttingen (integrierte Versorgung, keine Pflichtversorgung), des Niedersächsischen Landeskrankenhauses Göttingen (separierte Versorgung, Pflichtversorgung) und der Psychiatrischen Universitätsklinik Magdeburg (separierte Versorgung, Pflichtversorgung) aus. Die leitlinienkonformste Behandlung fand sich letztendlich in der Psychiatrischen Universitätsklinik Magdeburg, was, verkürzt formuliert, auf der Kombination von Separation und Universitätsstatus beruht [1,5-7]. Interessant war, dass bei der integrierten Versorgung in Göttingen im zeitlichen Verlauf eine Veränderung dahingehend stattfand, dass ältere Alte durch jüngere Alte quasi verdrängt wurden [1]. Bereits 2001 wies Gutzmann darauf hin, dass die Altersentwicklung in der stationären Psychiatrie dafür spricht, dass viele gerontopsychiatrische Patienten gar nicht erst in die stationäre Behandlung und möglicherweise „gleich ins Heim“ kommen [8].

Separation muss somit sein, wenn andernfalls die Interessen einer speziellen Patientengruppe untergehen. In diesem Sinne fordern Angehörigenvertreter, dass die Kompetenz für und das Interesse an Älteren (auf dem Praxisschild) erkennbar sein solle. Die Erfahrungen der Gerontopsychiatrie in Deutschland und in vielen anderen Ländern gehen jedoch in die andere Richtung. Trotz des Wissens um eine aufkommende Krise in der Versorgung psychisch kranker alter Menschen, trotz der Kenntnis, dass es viel zu wenig kompetente Fachleute im Bereich der Alterspsychiatrie und der Altersmedizin gibt, fand und findet sich kaum eine Bereitschaft, altersmedizinische und alterspsychiatrische Inhalte in die Aus- und Weiterbildungscurricula zu integrieren [9-11]. Schaut man sich die Hochschulen an, so muss man meinen, dass die Universitäten die bekannte Altersfeindlichkeit der Medizin immer noch zum Ausdruck bringen. Dabei ist das gesunde Altern auch ein Ergebnis des medizinischen Fortschritts und eine kulturelle Leistung [12].

Für eine Trennung der alterspsychiatrischen Versorgung sprechen also das umfangreiche Spezialwissen, die vorhandenen empirischen Daten und gesundheitspolitische Gründe. Erst eine selbstbewusst vertretene Alterspsychiatrie kann die Schnittstelle zur Allgemeinmedizin, Geriatrie und Neurologie angemessen „bedienen“. Erst in einem zweiten Schritt können dann meines Erachtens notwendige Modifikationen diskutiert werden. Hierzu könnte z.B. gehören, altersübergreifende Depressionsstationen anzubieten oder auch spezielle Konzepte für altgewordene chronisch psychisch Kranke zu entwickeln. Eine weitere Marginalisierung des Faches oder gar eine Reduktion auf eine Demenzpsychiatrie ist jedoch in keiner Weise zu vertreten.

Prof. Dr. med. Gabriela Stoppe

Universitäre Psychiatrische Kliniken

Wilhelm Klein-Straße 27

4025 Basel/Schweiz

E-mail: gabriela.stoppe@upkbs.ch

\section{Kontra}

Johannes Kipp

In unserer Gesellschaft wird es als ideal angesehen, wenn Alt und Jung zusammen wohnen und sich keine Altenghettos bilden [13]. Umso verwunderlicher ist es, dass in psychiatrischen Kliniken alte und junge Patienten voneinander abgesondert werden sollen, wobei unklar bleibt, zu wessen Heil und Nutzen. Das Milieu einer gerontopsychiatrischen Station mit älteren depressiven $\mathrm{Pa}$ tienten und unruhigen krankenhausbehandlungsbedürftigen Demenzpatienten ist sicher nicht erstrebenswert.

Aus unserer, in langjähriger Praxis bewährten Sicht entsteht ein günstiges Milieu auf psychiatrischen Stationen dann, wenn Patienten aller Diagnosegruppen dort gemeinsam untergebracht sind, deren Zusammenleben im Sinne einer Stationsgemeinschaft organisiert wird. Patienten, die ein krankheitsspezifisches störendes Verhalten zeigen, sollten zahlenmäßig kein Übergewicht bekommen. Werden beispielsweise mehr als zwei unruhige Demenzkranke auf einer Station untergebracht, so ist die Atmosphäre auf der Station meist schwer auszuhalten. Zur Zufriedenheit der Patienten trägt bei, wenn bei der Belegung der Zimmer darauf geachtet wird, dass die Zimmergenossen voraussichtlich gut miteinander kommunizieren können und sich wenig gegenseitig stören. Da viele ältere Patienten früher als jüngere ins Bett gehen, sollten beispielsweise solche Störmomente bei der Zimmerbelegung berücksichtigt werden.

Die psychiatrische Therapie ist in der Regel nicht an das Krankenbett gebunden. Eine gemeinsame Unterbringung heißt nicht, dass Patienten dann keine spezialisierten Therapien bekommen. Es ist wohl der Tradition der psychiatrischen Krankenhäuser als Heil- und Pflegeanstalten anzulasten, dass die Separierung psychisch Alterskranker von anderen psychisch Kranken als nützlich oder sinnvoll angesehen wird. Bisher gibt es keine Belege, dass dadurch ein adäquates Milieu zu erreichen wäre, in dem sich ältere Menschen wohl fühlen. Hohe Zwangseinweisungsraten, insbesondere bei Wiederaufnahmen [5], weisen auf das Gegenteil hin. Über die therapeutische Effektivität von separierenden Kliniken kann, da wissenschaftliche Untersuchungen fehlen, wenig gesagt werden. Die häufige Anordnung von typischen Neuroleptika (bei 77,1\% der Patienten in den Jahren 1998/99 [6]) in einer gerontopsychiatrischen Abteilung ist ein Hinweis, dass nicht nur deren Patienten, sondern auch deren Methoden als alt charakterisiert werden können.

\section{Beziehungsaufbau und Therapieangebote}

Wesentlich hängt das Milieu einer Station auch von den Beziehungen der Patienten zum Personal und den sich herstellenden Übertragungs- und Gegenübertragungsverhältnissen ab. Beim Besuch zahlreicher gerontopsychiatrischer Stationen traf der Autor außer in einer Abteilung, bei der schon 50-Jährige in die Gerontopsychiatrie aufgenommen wurden, auf Stationsmilieus, die durch Nichtkommunikation einerseits und durch teils fürsorgliche Hektik des Personals andererseits ausgezeichnet waren. Mitarbeiter, die ständig mit älteren psychisch kranken Menschen zu tun haben, können sich nicht besser und emotional wärmer auf ihre Patienten einlassen. Vielmehr besteht nach Einzelbeobachtungen bei ihnen die Gefahr, dass sie ein Burn-out-Syndrom entwickeln. 
Für alte Menschen sind spezialisierte, altersspezifische Therapien notwendig, da sich Lebenskrisen und Entwicklungsaufgaben von denen jüngerer Menschen unterscheiden. Außerdem sind die bestehenden körperlichen und psychischen Einschränkungen bei den Therapieangeboten zu berücksichtigen. Wir bieten beispielsweise sowohl täglich stattfindende Gruppentherapieprogramm für ältere Patienten mit Depressionen als auch für Patienten mit demenziellen Erkrankungen an [14]. Solche Gruppentherapieprogramme werden stationsübergreifend organisiert und können von den Patienten auch noch nach der Verlegung in die Tagesklinik oder nach der Entlassung ambulant besucht werden. Natürlich sind im Wohnbereich der Stationen auch Gruppenveranstaltungen von Alt und Jung notwendig, in denen es um die gemeinsame Gestaltung des Zusammenlebens geht. Separierte gerontopsychiatrische Tageskliniken sind dagegen sinnvoll, da es dort um Therapie geht und die Patienten durch die Behandlung nicht aus ihrer Lebenswelt herausgerissen werden.

\section{Qualifikation und Aufnahmeverpflichtung}

Durch die Bildung von spezialisierten gerontopsychiatrischen Einheiten (z. B. als Funktionsbereiche) besteht eine hohe Chance, dass die Mitarbeiter, insbesondere aber die Leiter, besondere Qualifikationen für ihre Aufgaben entwickeln, während in kleineren psychiatrischen Abteilungen die Mitarbeiter Allroundpsychiater sein müssen, die selten ein besonderes Fachwissen in der Gerontopsychiatrie erwerben. Dies kann jedoch nicht dafür ausschlaggebend sein, dass ältere Patienten separiert untergebracht werden müssen. Ein adäquater Kompetenzerwerb ist beispielsweise auch durch klinikinterne gerontopsychiatrische Arbeitsgruppen möglich.

Stationsgrößen sind meist durch bauliche Einheiten und organisatorische Vorgaben bestimmt. Hat eine Klinik eine sektorbezogene Aufnahmeverpflichtung, müssen jederzeit, - d.h. auch in der Nacht und am Wochenende - Betten für Notaufnahmen zur Verfügung stehen. Sind nur Spezialstationen vorhanden, kommen im Notdienst aufgenommene Patienten in die Stationen, in denen noch Betten frei sind. Dies bedeutet aber, dass sie im Akutzustand nicht spezialisiert behandelt werden und erst nach der Eingewöhnung in die aufnehmende Station nach wenigen Tagen in eine Spezialstation verlegt werden können, wo sie sich erneut eingewöhnen müssen. Verlegungen sind aber gerade bei älteren Patienten auch kritisch zu betrachten - einen älteren Baum verpflanzt man nicht.

Bei der integrativen Versorgung sind alle Stationen auch Aufnahmestationen, sodass hier von vornherein eine hohe Aufnahmekapazität besteht und Verlegungen nicht notwendig werden, was sich positiv auf die Kontinuität der Therapeut-Patienten-Beziehung auswirkt. Bei Wiederaufnahmen wird darauf geachtet, dass Patienten, die früher schon einmal auf einer bestimmten Station behandelt worden sind, dort wieder aufgenommen werden.

\section{Zusammenfassung}

Die integrative Versorgung psychisch Alterskranker geht davon aus, dass alte und junge Menschen mit unterschiedlichen Störungen auf den psychiatrischen Stationen zusammenleben. Bei Belegung der einzelnen Zimmer wird darauf geachtet, dass die Patienten zueinander passen. Bei der integrativen Versorgung lassen sich stationsübergreifende störungs- und altersspezifische Gruppentherapieprogramme sehr gut realisieren. Sie sind notwendig, da Therapievoraussetzungen, Therapieziele, Lebensaufgaben und vorhandene Einschränkungen körperlicher oder psychischer Art besondere Therapieangebote erforderlich machen. Die integrative Versorgung ermöglicht die Bildung lebendiger, kreativer Stationsmilieus, in denen Selbsthilfe und gegenseitige Hilfe gefördert werden, wenn es gelingt, dass nicht Patienten mit einer Störungsausprägung die gesamte Station in ihrer Kommunikation dominieren.

Dr. med. Johannes Kipp

Ludwig-Noll-Krankenhaus

Dennhäuser Straße 156

34134 Kassel

E-mail: Johannes.Kipp@Klinikum-Kassel.de

Literatur

${ }^{1}$ Stoppe G, Otto A, Koller M, Staedt J. Verlaufsuntersuchung zur gerontopsychiatrischen Behandlung im Vergleich zwischen integrierter Versorgung an einer Universität und separierter Versorgung an einem Landeskrankenhaus. I. Patientencharakteristik. Psychiat Prax 2005; 32: $239-244$

${ }^{2}$ Aupperle PM, MacPhee ER, Coyne AC, Blume J, Sanchez B. Health service utilization by Alzheimer's disease patients: a 2-year follow-up of primary versus subspecialty care. J Geriatr Psychiatry Neurol 2003; 16: $15-17$

${ }^{3}$ Wolfs CA, Dirksen CD, Severens JL, Verhey FR. The added value of a multidisciplinary approach in diagnosing dementia: a review. Int J Geriatr Psychiatry 2006; 21: 223-232

${ }^{4}$ Maercker A. Alterspsychotherapie. Aktuelle Konzepte und Therapieaspekte. Psychotherapeut 2003; 48: 132 - 149

${ }^{5}$ Staedt J, Sparfeld F, Otto A, Stoppe G. Stationäre Gerontopsychiatrische Behandlung - ein Vergleich zwischen integrierter versus separierter Versorgung an psychiatrischen Universitätskliniken. Teil 1: Patientencharakteristik. Psychiat Prax 2003; 30: $242-247$

${ }^{6}$ Staedt J, Sparfeld F, Otto A, Stoppe G. Stationäre Gerontopsychiatrische Behandlung - ein Vergleich zwischen integrierter versus separierter Versorgung an psychiatrischen Universitätskliniken. Teil 2: Diagnosen und Behandlung. Psychiat Prax 2003; 30: 248-255

${ }^{7}$ Stoppe G, Otto A, Koller M, Staedt J. Verlaufsuntersuchung zur gerontopsychiatrischen Behandlung im Vergleich zwischen integrierter Versorgung an einer Universität und separierter Versorgung an einem Landeskrankenhaus. II. Diagnosen und Behandlung. Psychiat Prax 2005; 32: $245-251$

${ }^{8}$ Gutzmann HH. Psychisch kranke alte Menschen: Wo sind sie geblieben? Spektrum 2001; 2: $31-33$

9 Jeste DV, Alexopoulos GS, Bartels SJ et al. Consensus statement on the upcoming crisis in geriatric mental health: research agenda for the next 2 decades. Arch Gen Psychiatry 1999; 56: 848-853

${ }^{10}$ Camus V, Katona C, Mendonca Lima CA de et al, on behalf of the WPA section on old age psychiatry. Teaching and training in old age psychiatry: a general survey of the World Psychiatric Association member societies. Int J Geriatr Psychiatry 2003; 18: 694-699

${ }^{11}$ Graham N, Lindesay J, Katona C et al, on behalf of the old Age Psychiatry section, World Psychiatric Association, World Health Organization. Reducing stigma and discrimination against older people with mental disorders: a technical consensus statement. Int J Geriatr Psychiatry 2003; 18: 670-678

12 Staehelin HB, Stoppe G. Die Medizin und das Alter: Fördert die Geriatrie die Akzeptanz des Älterwerdens? In: Bachmaier H (Hrsg): Die Zukunft der Altersgesellschaft. Analysen und Visionen. Göttingen: Wallstein, 2005: 87-98

${ }^{13}$ Ziller H. Altenhilfestrukturen der Zukunft - neue Wohnformen im Alter. Psychotherapie im Alter, 2006; 3 (3): im Druck

${ }^{14}$ Kipp J, Jüngling G. Einführung in die praktische Gerontopsychiatrie. 4. Aufl. München: Reinhardt, 2006 